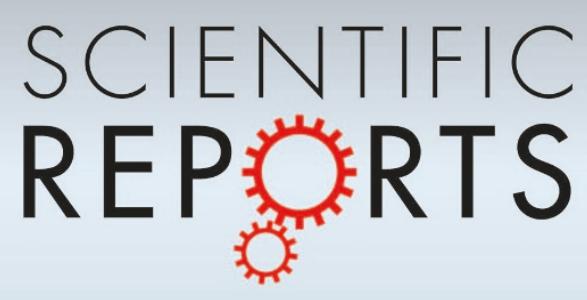

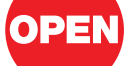

SUBJECT AREAS:

TERAHERTZ OPTICS

ELECTRONIC PROPERTIES AND

DEVICES

NANOPHOTONICS AND

PLASMONICS

OPTICAL PROPERTIES AND

DEVICES

Received

12 March 2013

Accepted

10 May 2013

Published

30 May 2013

Correspondence and requests for materials should be addressed to

J.G.

(jacek_gosciniak@ sutd.edu.sg)

\section{Theoretical investigation of graphene-based photonic modulators}

\author{
Jacek Gosciniak \& Dawn T. H. Tan
}

Engineering Product Development, Singapore University of Technology and Design, Singapore, 138682.

Integration of electronics and photonics for future applications requires an efficient conversion of electrical to optical signals. The excellent electronic and photonic properties of graphene make it a suitable material for integrated systems with extremely wide operational bandwidth. In this paper, we analyze the novel geometry of modulator based on the rib photonic waveguide configuration with a double-layer graphene placed between a slab and ridge. The theoretical analysis of graphene-based electro-absorption modulator was performed showing that a $3 \mathrm{~dB}$ modulation with $\sim 600 \mathrm{~nm}$-long waveguide is possible resulting in energy per bit below $1 \mathrm{fJ} / \mathrm{bit}$. The optical bandwidth of such modulators exceeds $12 \mathrm{THz}$ with an operation speed ranging from $160 \mathrm{GHz}$ to $850 \mathrm{GHz}$ and limited only by graphene resistance. The performances of modulators were evaluated based on the figure of merit defined as the ratio between extinction ratio and insertion losses where it was found to exceed 220.

$\mathrm{n}$ recent years it has become evident that bandwidth-limited electrical interconnects can no longer meet the growing demand on data processing and telecommunication traffic. Besides the limited capacity, electrical wires suffer from large energy consumption, signal attenuation and significant operational costs as interconnects densities rise. As opposed to it, photonics enables huge amounts of data to be moved at very high speeds with extremely low power over very small optical waveguides. One of the key components of the future telecommunication networks are optical modulators which serve as the gateway from the electrical to the optical domain. These are particularly attractive for low energy transmitters because they do not have a threshold that could limit the minimum operating energy and they may be easier to integrate with the available silicon platform ${ }^{1-3}$. An optical modulator can modify the properties of light such as its phase, amplitude or polarization by thermo-optic- ${ }^{4}$, electro-optic- ${ }^{5}$, or electro-absorption modulation ${ }^{6}$ and they are usually based on interference (Mach-Zehnder interferometers) ${ }^{7}$, resonance (ring resonators) ${ }^{8}$ and bandgap absorption (germanium-based electro-absorption modulators) ${ }^{9}$. However, they suffer either from slow switching time (thermo-optic switches), narrow operating bandwidth or large footprint (electro-optic modulators). Therefore, there is a need to find new technology which will enable high operation conditions over a small active region. The unique properties of graphene such as strong coupling with light, high-speed operation, and gate-variable optical conductivity ${ }^{10}$ make it a very promising material for realizing novel modulators ${ }^{11,12}$. Graphene offers the highest intrinsic mobility and the largest current density of any material, as well as an extraordinary thermal conductivity. These features make graphene ideal for use in the field of nanoelectronics. A single atom layer of graphene can provide the highest saturable absorption for a given amount of material - a phenomenon which enables highly efficient electro-absorption modulators ${ }^{13}$, photodetectors ${ }^{14}$ and power monitors ${ }^{15}$ to be realized.

Recently, the broadband electro-absorption modulator based on interband absorption of graphene was demonstrated with an overall length of $40 \mu \mathrm{m}$ and with a modulation depth of $0.16 \mathrm{~dB} / \mu \mathrm{m}$. This was achieved by placing a double-layer graphene on top of a silicon waveguide ${ }^{16}$. In this configuration, the effect of graphene conductivity change is not very pronounced as graphene is placed far from the electric field maximum of the propagating mode. In order to increase the effects of changes in graphene's conductivity on the propagating mode, the graphene should be placed at the maximum of the electric field ${ }^{17}$. It has been previously shown that plasmonics ridge waveguides ${ }^{18}$ are ideal candidates to realize graphene-assisted optical modulators since the electric field reaches its maximum at the interface between metal and dielectric ${ }^{13}$. Consequently, placing a doublelayer graphene between metal and dielectric will have strong effect on the propagating mode. Another concept is based on a ridge-type photonic modulator which consists of dual graphene layers separated by thin hexagonal boron nitride $(\mathrm{hBN})$ spacers placed at the center of the waveguide with maximum light intensity ${ }^{11}$. Although the concept is straightforward and obvious, fabrication of such structures possesses many challenges in terms of alignment, and hence, the technological imperfection might influence the resultant propagation characteristic of the mode. Compared to this configuration, the graphene-based modulator presented in this paper (Fig. 1(a)-(b)) 
(a)

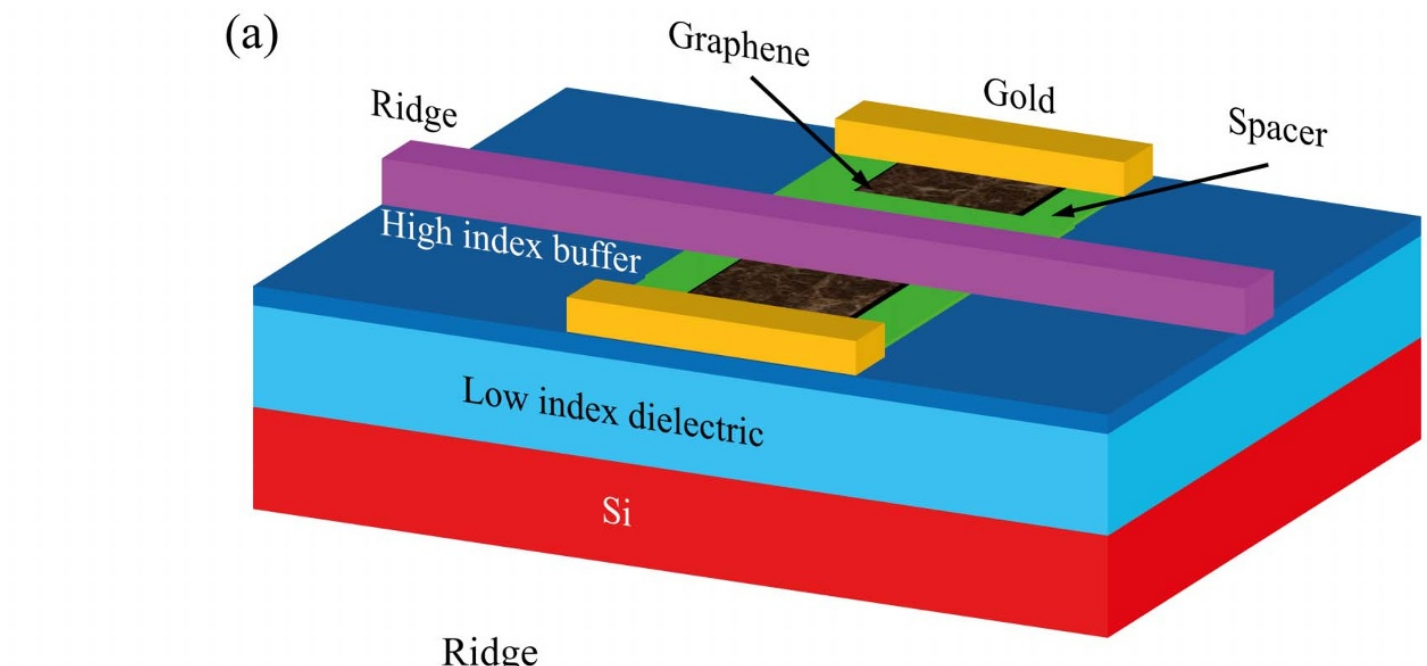

(b)

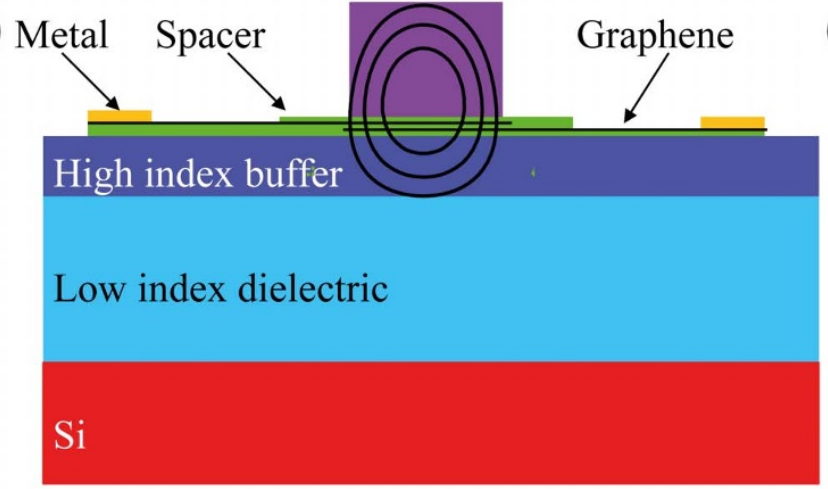

(c)

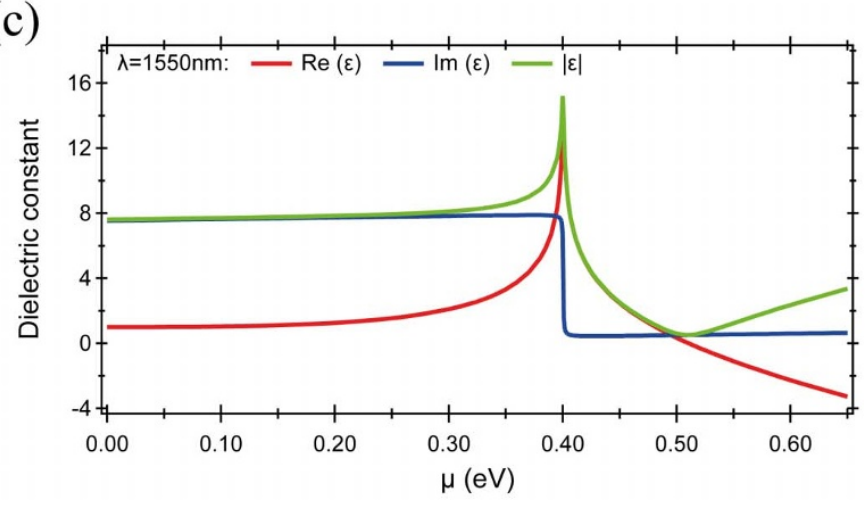

Figure $1 \mid$ Modulator configuration. (a) Schematic representation and (b) cross-section of the rib waveguide structure with a dielectric ridge on top of a double-layer graphene and dielectric spacers on the high-index buffer dielectric (slab) with the characteristic mode field distribution (black lines). The whole structure is supported by a low-index dielectric deposited on the Si wafer. (c) Calculated dielectric constant of graphene sheet (real part, imaginary part and magnitude) as a function of chemical potential for $\lambda=1550 \mathrm{~nm}$.

is based on the rib waveguide platform with the graphene sheets and spacers placed between waveguide and slab. Thus, the difficulties in alignment between top and bottom ridge region are avoided. Additionally, the rib waveguide approach provides some significant advantages compared to strip waveguides as it allows for enhanced flexibility and compatibility with all processing modules such as photodiodes and multiplexers.

\section{Results}

Geometry and gate-variable dielectric permittivity of graphene. The proposed double-layer graphene optical modulator is based on the rib waveguide configuration with the $\mathrm{Si}$ rib waveguide deposited on the buried-oxide layer. To maximize the influence of the graphene on the modulator performances, the double-layer graphene was placed between Si layer (slab) and Si ridge (Fig. 1(b)). The doublelayer graphene separated by a thin dielectric forms a simple parallel capacitor model in which the property is controlled by chemical potential $\mu$ and which can be tuned by electrical gating. Because of a synergy of the graphene properties induced by zero bandgap and symmetric valence and conduction bands, one graphene layer is doped by holes and the other by electrons at the same doping level. Thus, the applied gate voltage changes the charge-carrier density in graphene, $\mathrm{n}=\alpha\left(\mathrm{V}+\mathrm{V}_{0}\right)$, and accordingly shifts a Fermi level:

$$
E_{F}=\hbar v_{F} \sqrt{\pi \cdot n}
$$

where $V_{0}$ is the offset voltage caused by natural doping, $\alpha$ is estimated from a simple capacitor model $\left(\alpha=\varepsilon_{0} \varepsilon_{\mathrm{d}} / \mathrm{de}\right), \hbar$ is the Planck constant, $\mathrm{v}_{\mathrm{F}}$ is the Fermi velocity, and $\mathrm{n}$ is the electron/hole doping concentration.

The gate-dependent complex dielectric function of graphene, $\varepsilon(\omega)$, was obtained from the complex optical conductivity $\sigma(\omega)=$ $\sigma_{1}(\omega)+i \sigma_{2}(\omega)$ of graphene, consisting of interband and intraband contributions (Fig. 1(c)).

The dielectric constant of graphene varies very fast from $\varepsilon(0.4 \mathrm{eV})$ $=13.66+\mathrm{i} 6.71$ to $\varepsilon(0.7 \mathrm{eV})=-4.21+\mathrm{i} 0.69$ with a dip in the magnitude curve corresponding to $\mu=0.51$ where "dielectric graphene" is transforming to "metallic graphene" with $\varepsilon(0.51 \mathrm{eV})=$ $-0.50+\mathrm{i} 0.50$.

Position of graphene sheets. To investigate the performances of the modulator, different waveguide configurations were numerically analyzed with a double-layer graphene and dielectric spacer placed between slab and a ridge (Fig. 2). For all calculations the ridge width was kept constant at $\mathrm{w}=400 \mathrm{~nm}$ and sum of the ridge height and slab height at $\mathrm{h}=340 \mathrm{~nm}$, while changing only a ratio of the ridge height to the slab height. We started our calculations with the strip waveguide (ridge height: $\mathrm{h}=340 \mathrm{~nm}$, slab height: $\mathrm{t}=0 \mathrm{~nm}$ ) with a double-layer graphene and spacers placed on the top of the waveguide (Fig. 2(a)). For this configuration, the modulation depth of $0.18 \mathrm{~dB} / \mu \mathrm{m}$ and $0.037 \mathrm{~dB} / \mu \mathrm{m}$ for TM and TE mode was calculated to have good agreement with the experimental data of $0.16 \mathrm{~dB} / \mu \mathrm{m}^{16}$ obtained for the same configuration with the ratio of extinction ratio to insertion loss (ER-IL) 14.3 and 3.5 for TM and TE mode respectively. It should be noted that insertion losses were attributed in the paper to the propagation losses as the coupling 

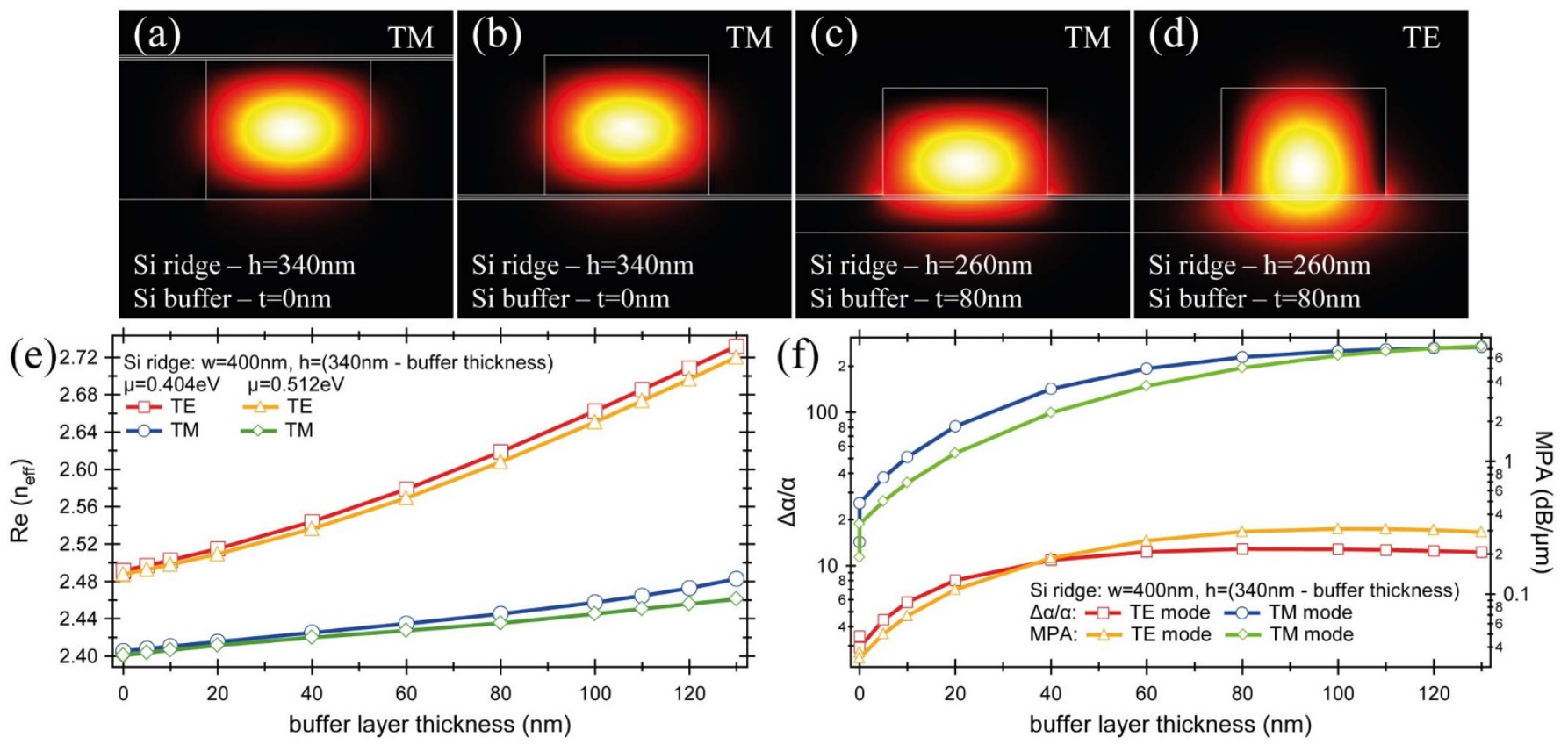

Figure $2 \mid$ Influence of the buffer layer thickness on the modulator performances. (a)-(d) Field distribution plot of the magnitude of the power flow for considered structure with a chemical potential $\mu=0.512 \mathrm{eV}$ for ridge waveguide configuration (a)-(b) with a double-layer on a top of the ridge (a), and below a ridge, between SOI and $\mathrm{Si}(\mathrm{b})$. Rib waveguide configuration (c)-(d) with a double-layer placed between slab and ridge for TM (c) and TE (d) modes.

losses from photonics waveguide to the modulator should be considered as being close to $0 \mathrm{~dB}$. For the same strip waveguide configuration, an improvement was observed for graphene and spacers placed below the ridge. i.e., between a buried-oxide and the $\mathrm{Si}$ ridge (Fig. 2(b)). For this configuration, the mode power attenuation (MPA) was calculated to be $0.34 \mathrm{~dB} / \mu \mathrm{m}$ (TM) and $0.033 \mathrm{~dB} / \mu \mathrm{m}$ (TE) with the ER-IL ratio of 25.5 (TM) and 2.9 (TE) respectively. Introducing a slab and reducing the ridge thickness moves the mode down, i.e., the maximum of the mode electric field is closer to the graphene sheet thus strengthening the interaction between them. For only a $40 \mathrm{~nm}$-thick slab and with the ridge thickness reduced from $340 \mathrm{~nm}$ to $300 \mathrm{~nm}$, the mode attenuation reaches $2.32 \mathrm{~dB} / \mu \mathrm{m}$ for the TM mode and $0.18 \mathrm{~dB} / \mu \mathrm{m}$ for the TE mode what translates to a significant improvement in the ER-IL ratio to 142 and 10.9 for the TM and TE modes respectively (Fig. 2(f)). However, even better performances was obtained for $80 \mathrm{~nm}$-thick slab and $260 \mathrm{~nm}$-thick ridge where the MPA was $5.05 \mathrm{~dB} / \mu \mathrm{m}$ for the TM mode and $0.29 \mathrm{~dB} / \mu \mathrm{m}$ for the TE mode with the calculated ER-IL ratio of 230 for TM mode and 12.8 for TE mode (Fig. 2(c)-(d)). Further increasing the slab thickness, while decreasing the ridge height, causes the mode to be pushed further into the slab resulting in weaker mode field confinement as the mode is spreads into the slab. Beyond a cutoff slab thickness of 100$110 \mathrm{~nm}$, a photonics mode is mostly supported in the slab layer.

It has to be emphasized that conventional GeSi electro-absorption modulators typically have ER-IL ratio which do not exceed 3.5. The optical modulators based on Si ridge waveguides integrated with graphene provide ER-IL ratios which are two orders of magnitude higher, offering a tremendous improvement over state of the art modulators.

TM/TE mode versus chemical potential. Based on the results from previous section, the detailed analysis was performed for a Si rib photonics waveguide with the slab thickness of $80 \mathrm{~nm}$ and with a $260 \mathrm{~nm}$-thick and $400 \mathrm{~nm}$-wide ridge and with a double-layer graphene and spacers placed between the slab and ridge. In the first step, the change in refractive index and MPA was analyzed as a function of chemical potential for different spacer dielectrics while keeping its thickness constant at $5 \mathrm{~nm}$. It has to be emphasized, that finding an appropriate dielectric spacer is another key issue which has to be addressed in obtaining efficient electro-absorption modulators. Firstly, good quality graphene sheets with high carrier mobility are formed on spacers with a small lattice mismatch with graphene. Secondly, very thin and high dielectric constant spacers are needed as it reduces the energy per bit and power consumption. Based on this, analyses were performed for two spacers with refractive indices of $n=1.98$ and $n=3.47$ corresponding to hexagonal boron nitride (hBN) and high- $\kappa$ dielectric respectively (Fig. 3(a)).

As shown in Fig. 3(a), a similar behavior in terms of the mode effective index and MPA is observed for both dielectric spacers. However, a few changes still can be observed in terms of the mode effective index which has strong effect on realization of the electrorefractive modulators. Firstly, the higher refractive index of spacers results in a slightly larger mode effective index so for $\mu=0 \mathrm{eV}$ it increases from $n_{\text {eff }}=2.353$ for the $\mathrm{hBN}$ spacer to $\mathrm{n}_{\text {eff }}=2.450$ for the high- $\kappa$ dielectric spacer. Secondly, for the modulator with the low- $\kappa$ dielectric spacer (hBN), the minimum mode effective index occurs at $\mu=0.495 \mathrm{eV}$ whereas for the high- $\kappa$ dielectric spacer it shifts to $\mu=$ $0.500 \mathrm{eV}$. At the same time, the maximum mode effective index is not affected by the spacer and remains at $\mu=0.530 \mathrm{eV}$. Apart from it, the difference between maximum and minimum mode effective index increases from $\Delta \mathrm{n}=0.118$ for the low- $\kappa$ dielectric spacer $(\mathrm{hBN})$ to $\Delta \mathrm{n}=0.144$ for the high- $\kappa$ dielectric spacer. In terms of a chemical potential, the change between minimum and maximum mode effective index is $\Delta \mu=0.035 \mathrm{eV}$ for the $\mathrm{hBN}$ spacer and $\Delta \mu$ $=0.030 \mathrm{eV}$ for the high- $\kappa$ dielectric spacer. However, to achieve the same charge carrier mobility in graphene, a lower voltage change is required for the high- $\kappa$ dielectric spacers. The required voltage change was calculated to be $\Delta \mathrm{V}=0.505 \mathrm{~V}$ for the $\mathrm{hBN}$ spacer while for the high- $\kappa$ dielectric spacer it drops more than four times to $\Delta \mathrm{V}$ $=0.141 \mathrm{~V}$. Additionally, to introduce a $\pi$-phase shift between both 

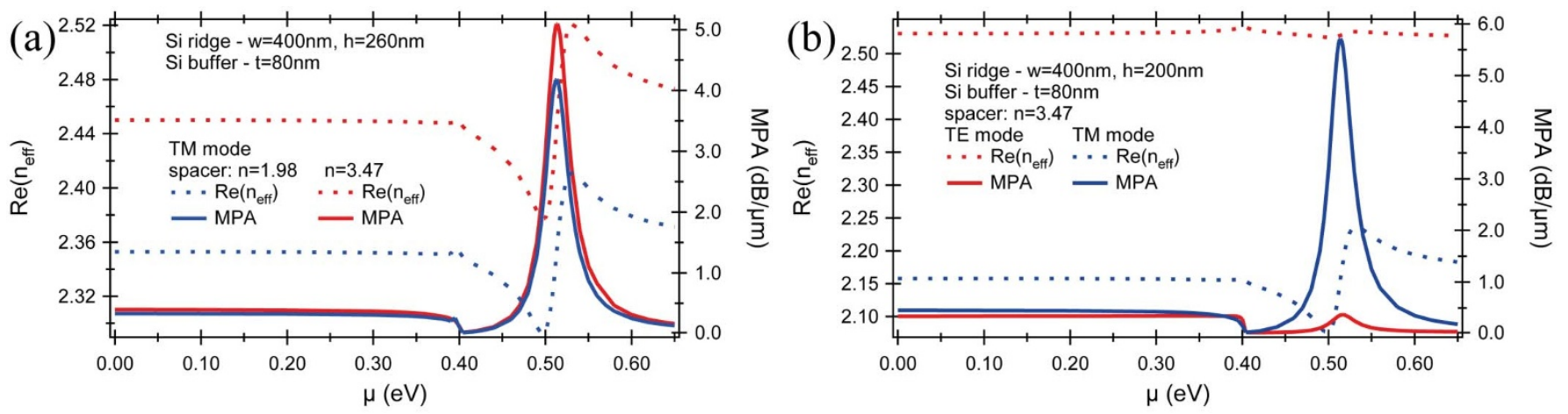

Figure $3 \mid$ Influence of the spacer on the TE/TM modes. (a) The mode effective index (real part) and MPA of the graphene-based photonics Si rib waveguide as a function of chemical potential at $\lambda=1550 \mathrm{~nm}$ for (a) two different spacers sandwiched between Si slab $(\mathrm{t}=80 \mathrm{~nm})$ and $\mathrm{Si}$ ridge $(\mathrm{w}=400 \mathrm{~nm}$ and $\mathrm{h}=260 \mathrm{~nm}$ ) and for (b) two supported modes - TM and TE for a configuration with the high- $\kappa$ dielectric spacer.

arms of the graphene-based Mach-Zehnder interferometer, an active arm length of $6.57 \mu \mathrm{m}$ is needed for structure with the $\mathrm{hBN}$ spacer and only $5.4 \mu \mathrm{m}$ with the high- $\kappa$ dielectric spacer.

For a $6.57 \mu \mathrm{m}$-long and $0.4 \mu \mathrm{m}$-wide modulator with the $\mathrm{hBN}$ spacer, the capacitance of the device was calculated to be $18 \mathrm{fF}$, which in tandem with the voltage change of $\Delta \mathrm{V}=0.505 \mathrm{~V}$ needed to ensure a full modulation results in an energy per bit on the order of $1.16 \mathrm{ff} / \mathrm{bit}$. Conversely, for a $5.4 \mu \mathrm{m}$-long and $0.4 \mu \mathrm{m}$-wide modulator with a high- $\kappa$ dielectric it was calculated to be only $\mathrm{E}_{\mathrm{bit}}=$ $0.23 \mathrm{fJ} / \mathrm{bit}$ with calculated capacitance of $46 \mathrm{fF}$ and $\Delta \mathrm{V}=0.141 \mathrm{~V}$.

Since the optical absorption in graphene can be controlled through electrical gating, the graphene can be used as the active medium in an optical electro-absorption modulator. Therefore, conductivity change induced by an applied gate voltage has a strong effect on the propagating mode (Fig. 3(a)). Regardless of the type of spacer material, the minimum absorption of $\sim 0.02 \mathrm{~dB} / \mu \mathrm{m}$ was observed for $\mu=0.404 \mathrm{eV}$ while for $\mu=0.512 \mathrm{eV}$ the absorption arises to the maximum of $\sim 4.172 \mathrm{~dB} / \mu \mathrm{m}$ and $\sim 5.052 \mathrm{~dB} / \mu \mathrm{m}$ for the $\mathrm{hBN}$ and high- $\kappa$ dielectric spacers respectively. Thus, to achieve a $3 \mathrm{~dB}$ modulation depth a $720 \mathrm{~nm}$-long waveguide is required for structure with the hBN spacer, and only a $595 \mathrm{~nm}$-long waveguide for structure with the high- $\kappa$ dielectric spacer. Furthermore, the efficiency of the electro-absorption modulator estimated by the figure of merit, $\Delta \alpha / \alpha$, shows slightly better performances with a high- $\kappa$ dielectric spacer where it was calculated to be 229 compared to 224 obtained for a low$\kappa$ dielectric spacer. Apart from the optical properties, the investigated graphene-based electro-absorption modulators possess excellent electrical properties such as the energy per bit consumption. The voltage required to switch modulator from its minimum absorption state to maximum was evaluated to be $\Delta \mathrm{V}=0.453 \mathrm{~V}$ and $\Delta \mathrm{V}=$ $1.394 \mathrm{~V}$ what corresponds to the $\mathrm{E}_{\mathrm{bit}}=0.26 \mathrm{fJ} / \mathrm{bit}$ for modulator with the $\mathrm{hBN}$ spacer and $\mathrm{E}_{\mathrm{bit}}=0.96 \mathrm{ff} / \mathrm{bit}$ with the high- $\kappa$ dielectric spacer respectively.

One of the ways to increase the modulator attenuation is to push mode down into a slab such that graphene resides closer to the mode field maximum. Thus, the interaction between mode field and a graphene is maximized. It can be achieved either by increasing the slab thickness or by decreasing the ridge dimensions. In our calculations the slab thickness and ridge width were kept constant while the ridge height was decreased from $260 \mathrm{~nm}$ to $200 \mathrm{~nm}$ (Fig. 3(b)). The analysis was performed for a high- $\kappa$ dielectric spacer and for both supported modes - TM and TE. It was found that, as the attenuation curves for both modes exhibit similar behavior, the attenuation of the TM mode is significantly larger than that of the TE mode, and the change of chemical potential is observed to have a stronger effect on the TM mode. Most of all, for $\mu=0.51 \mathrm{eV}$ there is a transition from "dielectric graphene" to "metallic graphene" corresponding to a dip in the curve of dielectric constant (Fig. 1(c)). Specifically, in the absence of applied voltage, $\mu=0 \mathrm{eV}$, the attenuation losses for both modes are similar with $0.34 \mathrm{~dB} / \mu \mathrm{m}$ for the TE mode and $0.46 \mathrm{~dB} / \mu \mathrm{m}$ for the TM mode respectively and this trend dominates up to $\mu \approx$ $0.4 \mathrm{eV}$. This is consistent with observed trends in silicon photonics, where TM polarization is less commonly used because propagation losses tend to be higher. For $\mu \approx 0.404 \mathrm{eV}$, the attenuation losses for both modes reach a minimum of $0.025 \mathrm{~dB} / \mu \mathrm{m}$ what is manifested by decreases in the imaginary part of graphene's dielectric constant and increase in its real part (Fig. 1(c)). As $\mu$ is further increased, the losses encountered in polarizing the graphene are keep constant in low level but the polarization strength induced by a mode and described by real part of dielectric constant drops very fast what increases the MPA of both modes. However, the attenuation of the TM mode is larger than that of TE mode. At $\mu=0.51 \mathrm{eV}$, the real part of dielectric constant becomes negative and the plasmonics mode associated with TM polarized light and propagating in the interface between graphene and dielectric emerges. As the negative value of the real part of dielectric constant arises, the absorption losses and MPA decrease. It should be emphasized that maximum absorption of the mode corresponds to a minimum value of the graphene dielectric constant i.e., a dip in the curve of dielectric constant magnitude ${ }^{17}$. This "epsilonnear-zero" effect can be seen almost in any material at its plasma frequency. However, compared to other materials, the uniqueness of graphene lies in that its plasma frequency can be tuned by electrical gating. Furthermore, the magnitude of dielectric constant varies more than 30 times between $\mu=0.4 \mathrm{eV}$ (maximum dielectric constant magnitude) and $\mu=0.51 \mathrm{eV}$ (minimum dielectric constant magnitude) what explains high modulation depth achieved with the graphene-based modulators.

Wavelength dependence of MPA, optical bandwidth. Apart from high modulation speed, small footprint and high modulation strength (efficiency), the novel integrated modulators require large optical bandwidth for applications in on-chip optical interconnects. However, due to the poor electro-optic properties of regular materials the conventional electro-optic modulators suffer either from very large footprint or narrow bandwidth. In comparison to compound semiconductors, the ultrahigh carrier mobility and optical absorption of graphene which is independent of wavelength, enables a new optical modulators with ultra-broad optical bandwidth.

To evaluate the bandwidth of the presented rib waveguide photonic modulator, the dependence of chemical potential on graphene's dielectric constant and, in consequence, on the mode effective index and MPA was studied for different wavelengths covering the entire telecommunications bandwidth. As shown in Fig. 4(a)-(b), the graphene's dielectric constant and consequently the photonics mode vary with the wavelength. Increases in the chemical potential causes a peak in the real part of dielectric constant (Fig. 4(a)), corresponding 

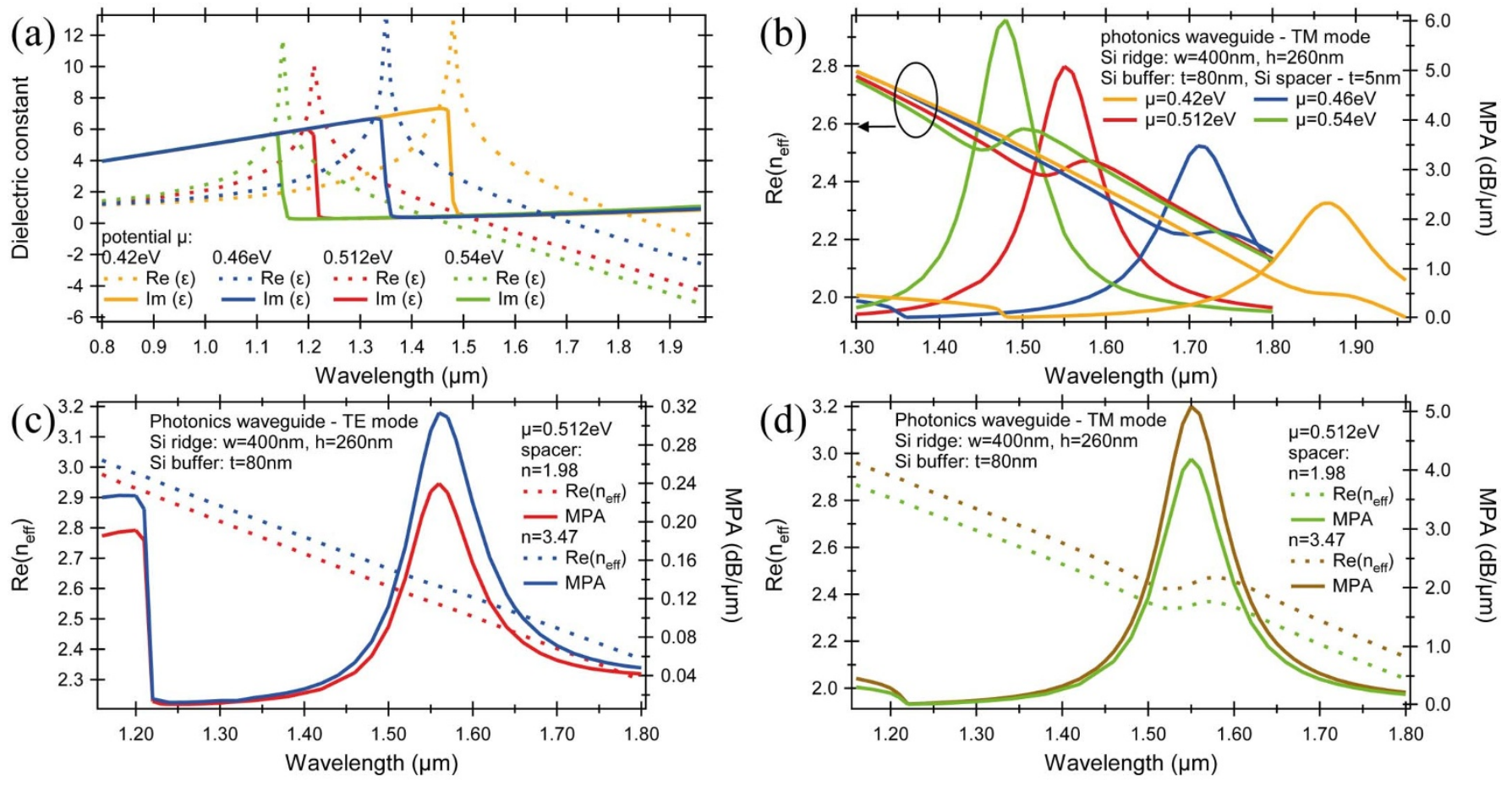

Figure $4 \mid$ Wavelength dependence on the modulator performances. (a) Calculated dielectric constant of graphene sheet (real part and imaginary part) as a function of wavelength for different chemical potentials at $\lambda=1550 \mathrm{~nm}$ with (b) a corresponding mode effective index and MPA for TM propagating mode. (c)-(d) Mode effective index and MPA as a function of wavelength for different spacer materials for (c) TE and (d) TM modes.

to the minimum MPA, and a dip in the magnitude of dielectric constant (transformation of "dielectric" graphene to "metallic" graphene), corresponding to the maximum MPA, move to the lower wavelengths which has direct influence on the MPA which redshift as well (Fig. 4(b)).

Thus, for a chemical potential of $0.512 \mathrm{eV}$, the dip in the magnitude of dielectric constant is observed at a wavelength of $1550 \mathrm{~nm}$, what fulfill the requirements of maximum losses of the modulator. Away from the central wavelength, the MPA decreases. Consequently, the wavelength spanning from $1520 \mathrm{~nm}$ to $1580 \mathrm{~nm}$ only results in a decrease in modulation depth of $\sim 1.5 \mathrm{~dB} / \mu \mathrm{m}$.

Consequently, for a lower potential, $\mu=0.46 \mathrm{eV}$, the dip in the real part of the dielectric constant moves towards longer wavelengths with a maximum MPA corresponding to $\lambda \approx 1710 \mathrm{~nm}$. Thus, for a $1 \mu \mathrm{m}$-long modulator, a $3 \mathrm{~dB}$ optical bandwidth was calculated to be 14.1 THz near $1550 \mathrm{~nm}, 12.3 \mathrm{THz}$ near $1480 \mathrm{~nm}$ and $13.6 \mathrm{THz}$ near $1710 \mathrm{~nm}$ for a chemical potential of $0.512 \mathrm{eV}, 0.54 \mathrm{eV}$ and $0.46 \mathrm{eV}$ respectively. For a lower chemical potential, $\mu=0.42 \mathrm{eV}$, a $3 \mathrm{~dB}$ modulation requires at least $1.5 \mu \mathrm{m}$-long waveguide with the optical bandwidth calculated for a $2 \mu \mathrm{m}$-long modulator exceeding $15.1 \mathrm{THz}$.

Apart from the chemical potential, the wavelength dependence of the MPA and mode effective index was studied for different spacer materials and for both supported modes - TE and TM (Fig. 4(c)(d)). For a high- $\kappa$ dielectric spacer and for the modulator length of $1 \mu \mathrm{m}$, the $5.08 \mathrm{~dB}$ mode attenuation can be achieved while for a low$\kappa$ dielectric spacer the same level of mode attenuation requires at least a $1.2 \mu \mathrm{m}$-long interaction length. The optical bandwidth for both modulators was calculated at $14.1 \mathrm{THz}$ with a central wavelength at $1550 \mathrm{~nm}$. Based on this it can be concluded that spacer not affect an optical bandwidth of the modulator but it impacts the modulator length (Fig. 4(d)).

For the TE supported mode (Fig. 4(c)) with a mode attenuation of $5.08 \mathrm{~dB}$, the interaction length of $16.2 \mu \mathrm{m}$ is required for structure with a high- $\kappa$ dielectric spacer and $21.2 \mu \mathrm{m}$ for a low- $\kappa$ dielectric spacer. Additionally, the central wavelength corresponding to the maximum mode attenuation shifts for a TE mode from $1550 \mathrm{~nm}$ to $1560 \mathrm{~nm}$ with a $3 \mathrm{~dB}$ bandwidth calculated to be $16.5 \mathrm{THz}$ for both dielectric spacers.

Therefore, this modulator shows broadband operation so hundreds of channels from different systems can be processed in the same device because of weak wavelength dependence.

Modulation speed. Due to the exceptionally high carrier mobility and high saturation velocity, the operation bandwidth is not likely limited by the carrier transit time. The relaxation time is inversely proportional to the degree of crystalline disorder in the graphene so with a high quality of graphene it can operates on the timescale of picoseconds, which implies that graphene-based electronics may operate at $500 \mathrm{GHz}$. In practice, the maximum operating bandwidth is limited by the RC constant of the device.

As the calculated capacitance is very low for both dielectric spacers, the main limiting factor of the capacitive delay is graphene resistance with the graphene sheet resistance of $23.5 \Omega / \square$ for $\mu=$ $0 \mathrm{eV}$. Thus, for modulator working in a low loss regime, i.e., small losses for OFF voltage state, to obtain a $3 \mathrm{~dB}$ modulation it requires to introduce a modulator to a high loss regime, which is achieved by increasing the chemical potential. For a $0.72 \mu \mathrm{m}$-long modulator with a low- $\kappa$ dielectric spacer $(\varepsilon=3.9)$, a $3 \mathrm{~dB}$ modulation is achieved with an operation speed of $0.51 \mathrm{THz}$ while keeping an energy per bit at a low level of $0.96 \mathrm{fJ} / \mathrm{bit}$. Conversely, for modulator with a high- $\kappa$ dielectric spacer, the active length of around $0.595 \mu \mathrm{m}$ is sufficient to achieve a $3 \mathrm{~dB}$ modulation with an operation speed of $0.16 \mathrm{THz}$ and $\mathrm{E}_{\mathrm{bit}}=0.26 \mathrm{fJ} / \mathrm{bit}$. Thus, it can be confirmed there is fundamental energy-speed tradeoff. Additionally, it should be noted that adaptation of longer modulators will not affect the modulation speed but will improve the modulation depth which will be however, at the cost of $\mathrm{E}_{\mathrm{bit}}$. Furthermore, it can be summarized that low- $\kappa$ dielectric spacers offer a higher operation speed but at the cost of energy and footprint (Fig. 3(a)).

To go beyond a $\sim 1 \mathrm{THz}$ operation speed, the modulator should be introduced in a high loss regime with OFF applied voltage state 
corresponding to the maximum modulation depth for $\mu=0.512 \mathrm{eV}$ (Fig. 3(a)). A $3 \mathrm{~dB}$ modulation for a $0.72 \mu \mathrm{m}$-long waveguide with a low- $\kappa$ dielectric spacer requires significant increases in the chemical potential which can be realized by increases in the bias voltage. At the same time, with increases in the bias voltage, the graphene conductivity arises and graphene sheet resistance decreases resulting in an increase in the operation speed. Thus, for $\mu=1.0 \mathrm{eV}$ corresponding to voltage increases to $14 \mathrm{~V}$, the bandwidth increases to $3.5 \mathrm{THz}$ at the cost of the energy per bit which increases to $69 \mathrm{fJ} / \mathrm{bit}$. Compared to it, with a high- $\kappa$ dielectric spacer and for a modulator length of $0.595 \mu \mathrm{m}$, the bandwidth drops to $1.14 \mathrm{THz}$ with $\mathrm{E}_{\mathrm{bit}}=18 \mathrm{fJ} / \mathrm{bit}$ for $\mu=1.0 \mathrm{eV}$ corresponding to voltage of $4.5 \mathrm{~V}$.

\section{Discussion}

In summary, we propose novel graphene-based photonics electroabsorption modulators based on a rib waveguide configuration. To maximize the influence of graphene on the modulator performance, the double-layer graphene was placed between a rib slab and a ridge, close to the mode field maximum. In this configuration, the conductivity of graphene was dynamically tuned by a gate voltage with a strong effect on the propagating mode. Regardless of the graphene position, it was found that TM mode gives better modulation ability than the TE mode with a modulation depth calculated to be $0.18 \mathrm{~dB} /$ $\mu \mathrm{m}$ and $0.037 \mathrm{~dB} / \mu \mathrm{m}$ for TM and TE mode respectively and for the modulator configuration with graphene placed on top of the waveguide. The figure of merit in this configuration was 14.3 for the TM mode and 3.5 for TE mode. Significant improvement was observed for graphene placed between $80 \mu \mathrm{m}$-thick slab and $260 \mu \mathrm{m}$-thick ridge with a figure of merit arising to 230 for the TM mode and 12.8 for the TE mode. Additionally, the influence of the spacer on the overall performance of the modulator was considered showing that with high refractive indices spacers the modulator length can be reduced from $720 \mathrm{~nm}$ for the hBN spacer to $595 \mathrm{~nm}$ for the high- $\kappa$ dielectric spacer. As optical interconnects require large optical bandwidth, the wavelength dependence of the mode attenuation was studied for different chemical potentials showing an optical bandwidth exceeding $14 \mathrm{THz}$.

Apart from the optical properties, the electrical properties of the modulator were studied in terms of the energy per bit and operation speed. It was confirmed that there is a fundamental tradeoff between energy consumption and speed. For the hBN spacer, a $3 \mathrm{~dB}$ modulation was achieved with an operation speed of $0.51 \mathrm{THz}$ and with energy consumption of $0.96 \mathrm{fJ} / \mathrm{bit}$ whereas for a high- $\kappa$ dielectric spacer it was found to be $0.16 \mathrm{THz}$ and $0.26 \mathrm{fJ} / \mathrm{bit}$ respectively.

As it was shown, the presented configuration enables realization of very efficient modulators with a nanoscale footprint, small losses and with huge optical/electrical bandwidth for a future on-chip optical interconnects.

\section{Methods}

The proposed modulator geometry was investigated using two-dimensional finite element method (FEM) simulations at the telecom wavelengths using commercial software COMSOL. The FEM is a well know technique for numerical solution of partial differential equations or integral equations, where the region of interest is subdivided into small segments and the partial differential equation is replaced with a corresponding functional. In the calculations, the refractive indexes of the $\mathrm{Si}$ rib, the $\mathrm{SiO}_{2}$ buffer and the spacers are $\mathrm{n}_{1}=3.47, \mathrm{n}_{2}=1.444$ and $\mathrm{n}_{3}=1.98$ for low- $\mathrm{\kappa}$ dielectric spacers and $\mathrm{n}_{4}=3.47$ for high- $\kappa$ dielectric spacers, respectively

To evaluate the mode effective index and mode power attenuation of the considered structure the gate-dependent complex dielectric constant of graphene has to be calculated. The complex dielectric function $\varepsilon(\omega)$ can be obtained from the complex optical conductivity of graphene, consisting of interband and intraband contributions,

$$
\begin{gathered}
\sigma_{\text {total }}=\sigma_{\text {intra }}+\sigma_{\text {inter }}^{\prime}+i \sigma_{\text {inter }}^{\prime \prime} \\
\sigma_{\text {ntra }}=\sigma_{0} \frac{4 \mu}{\pi} \frac{1}{\hbar\left(\tau_{1}-i \omega\right)}
\end{gathered}
$$

$$
\begin{gathered}
\sigma_{\text {inter }}^{\prime}=\sigma_{0}\left(1+\frac{1}{\pi} \arctan \frac{\hbar \omega-2 \mu}{\hbar \tau_{2}}-\frac{1}{\pi} \arctan \frac{\hbar \omega+2 \mu}{\hbar \tau_{2}}\right) \\
\sigma_{\text {inter }}^{\prime \prime}=-\sigma_{0} \frac{1}{2 \pi} \ln \frac{(2 \mu+\hbar \omega)^{2}+\hbar^{2} \tau_{2}^{2}}{(2 \mu-\hbar \omega)^{2}+\hbar^{2} \tau_{2}^{2}}
\end{gathered}
$$

where $\sigma_{0}=\mathrm{e}^{2} /(4 \hbar)=60.8 \mu \mathrm{S}$ is the universal optical conductance, by using:

$$
\varepsilon(\omega)=1+\frac{i \sigma(\omega)}{\omega \varepsilon_{0} \mathrm{~d}_{g}},
$$

where, $\mathrm{d}_{\mathrm{g}}=0.7 \mathrm{~nm}$ is a thickness of the graphene layer.

As the complex conductivity $\sigma(\omega, \mu, \Gamma, T)$ depends on the angular frequency $\omega$, the charge particle scattering rate $\Gamma=1 / \tau$ with $\tau$ being the relaxation time, the chemical potential $\mu$ and temperature $\mathrm{T}^{19}$, the dielectric constant of the graphene layer was calculated as a function of chemical potential for $\lambda=1550 \mathrm{~nm}, \mathrm{~T}=296 \mathrm{~K}$ (room temperature) and $\tau_{1}=1.2 \mathrm{ps}$ for interband conductivity and $\tau_{2}=10 \mathrm{fs}$ for intraband conductivity.

1. Shacham, A., Bergman, K. \& Carloni, L. P. Photonic network-on-chip for future generations of chip multiprocessors. IEEE Trans. Comput. 57, 1246-1260 (2008).

2. Miller, D. A. B. Devices Requirements for Optical Interconnects to Silicon Chips. Proceedings of the IEEE 97, 1166-1185 (2009).

3. Tan, D. T. H. et al. Wide bandwidth, low loss 1 by 4 wavelength division multiplexer on silicon for optical interconnects. Opt. Express 19, 2401-2409 (2011).

4. Coppola, G., Sirleto, L., Rendina, I. \& Iodice, M. Advance in thermo-optical switches: principles, materials, design, and device structure. Opt. Engineering, 50, 071112 (2011).

5. Soref, R. \& Bennett, B. Electrooptical effects in silicon. IEEE J. Quant. Electron. 23, 123-129 (1987).

6. Reed, G. T., Mashanovich, G., Gardes, F. Y. \& Thomson, D. J. Silicon optical modulators. Nature Photonics 4, 518-526 (2012).

7. Gosciniak, J., Markey, L., Dereux, A. \& Bozhevolnyi, S. I. Efficient thermooptically controlled Mach-Zehnder interferometers using dielectric-loaded plasmonic waveguides. Opt. Express 20, 16300-16309 (2012).

8. Gosciniak, J. et al. Thermo-optic control of dielectric-loaded plasmonic waveguide components. Opt. Express 18, 1207-1216 (2010).

9. Feng, D. et al. High speed GeSi electro-absorption modulator at $1550 \mathrm{~nm}$ wavelength on SOI waveguide. Opt. Express 20, 22224-22232 (2012).

10. Wang, F. et al. Gate-variable optical transitions in graphene. Science 320, 206-209 (2008).

11. Kim, K., Choi, J.-Y., Kim, T., Cho, S.-H. \& Chung, H.-J. A role for graphene in silicon-based semiconductor devices. Nature 479, 338-344 (2011).

12. Novoselov, K. S. et al. A roadmap for graphene. Nature 490, 192-200 (2012).

13. Sensale-Rodriguez, B. et al. Efficient terahertz electro-absorption modulation employing graphene plasmonic structures. Appl. Phys. Lett. 101, 261115 (2012).

14. Xia, F., Mueller, T., Lin, Y. M., Valdes-Garcia, A. \& Avouris, P. Ultrafast graphene photodetector. Nat. Nanotechnol. 4, 839-843 (2009).

15. Gosciniak, J., Nielsen, M. G., Markey, L., Dereux, A. \& Bozhevolnyi, S. I. Power monitoring in dielectric-loaded plasmonic waveguides with internal Wheatstone bridges. Opt. Express 21, 5300-5308 (2013).

16. Liu, M., Yin, X. \& Zhang, X. Double-layer graphene optical modulator. Nano Lett. 12, 1482-1485 (2012)

17. Lu, Z. \& Zhao, W. Nanoscale electro-optic modulators based on graphene-slot waveguides. J. Opt. Soc. Am. B 29, 1490-1496 (2012).

18. Gosciniak, J. \& Tan, D. T. H. Graphene-based waveguide integrated dielectricloaded plasmonic electro-absorption modulators. Nanotechnology 24, 185202 (2013).

19. Bao, Q. \& Loh, K. P. Graphene photonics, plasmonics, and broadband optoelectronic devices. ACS Nano 6, 3677-3694 (2012).

\section{Acknowledgements}

Support from the SUTD-MIT International Design Center and SUTD-ZJU Collaboration Research Grant is gratefully acknowledged.

\section{Author contributions}

J.G. conceived the idea, designed the structures, performed theoretical calculations and FEM simulations and wrote the manuscript. D.T. supervised the project.

\section{Additional information}

Competing financial interests: The authors declare no competing financial interests.

License: This work is licensed under a Creative Commons Attribution 3.0 Unported License. To view a copy of this license, visit http://creativecommons.org/licenses/by/3.0/ How to cite this article: Gosciniak, J. \& Tan, D.T.H. Theoretical investigation of graphene-based photonic modulators. Sci. Rep. 3, 1897; DOI:10.1038/srep01897 (2013). 